REVISTA DE

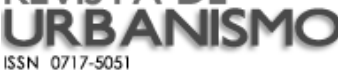

http://revistaurbanismo.uchile.cl
Manejo y gestión de áreas históricas. El Plan Maestro de La Habana Vieja 1995-96

Handling and management of historic areas. The Master Plan 1995-96 La Habana Vieja 1995-96

\title{
Manejo y gestión de áreas históricas. El Plan Maestro de La Habana Vieja 1995-96
}

Handling and management of historic areas. The Master Plan 1995-96 Old Havana.

Vico Muñoz Köhler

\section{Filiación}

Dipl. Ing. en Planificación Urbana y Regional, TU-Berlín, Técnico del Servicio Alemán de Cooperación Social-Técnica, actualmente en Ecuador en: Programa Nacional de Asesoramiento en la Construcción y Mejoramiento de la Vivienda Popular, CAVIP - Banco Ecuatoriano de la Vivienda, GTZ.

\section{Resumen}

Mucho ha sido dicho en La Habana acerca del manejo y la gestión de áreas históricas. Sin embargo, el caso cubano -a diferencia de otros casos- necesitará de un preámbulo de pasos complejos que deben ser resueltos antes de abordar la gestión de los barrios propiamente tal.

\section{Palabras clave}

Gestión de áreas históricas, La Habana Vieja.

\begin{abstract}
Much has been said in Havana about the handling and management of historic areas. However, the Cuban case -unlike others cases- need a preamble of complex steps that must be resolved before addressing the management of the districts itself.
\end{abstract}

\section{Key words}

Management of historic areas, Old Havana.

\section{Sumario}

Introducción

Dos políticas macro-regionales Cuba - RDA

Presentación del Plan Maestro de La Habana Vieja

Desarrollo urbano y problemas principales generados

Los desafíos

Zonificación de La Habana Vieja

¿Cómo generar recursos para financiar obras barriales?

Capacidad de gestión: dinámica participativa

Planos 


\section{Introducción}

La Habana, capital de la República de Cuba, fue una de las siete primeras villas fundadas durante la colonización hispana de la isla, después del "descubrimiento" de C. Colón (1519). Ciudad fortificada, fue considerada entre las más importantes plazas fuertes de América, acumulando durante cuatro siglos obras arquitectónicas y artísticas de gran valor que le confieren, en 1982, el reconocimiento de "Patrimonio de la Humanidad" de la UNESCO.

Dentro del patrimonio cultural hispano-Iusitano-americano, La Habana Vieja es uno de los barrios históricos "vivos" densamente poblados más atractivos e interesantes, incluyendo ejemplos de la Península Ibérica.

\section{Dos políticas macro-regionales Cuba - RDA}

Ya veinte años atrás, el gobierno cubano promueve planes de impacto regional tendientes al desarrollo equilibrado de los centros poblados y para evitar que la capital, La Habana, crezca desproporcionadamente debido a una inmigración descontrolada.

Con la mira puesta sobre el proceso de la "descentralización", se invierte principalmente en la infraestructura regional, para conferirle "atracción" centrípeta a las pequeñas y medianas ciudades mediante la realización de proyectos productivos descentralizados y proyectos de interés social como centros comerciales, de cultura, deportes y esparcimiento.

Contrastando con ello, en la República Democrática Alemana / RDA (DDR), se procede a la inversa. Los dos Berlín: "la isla Berlín Occidental", como "escaparate del capitalismo" enclavado dentro de su territorio, y "Berlín Oriental - capital de la RDA", rivalizan agresivamente, generando en el gobierno oriental planes que, ante todo, tienden con gran esfuerzo al desarrollo de la infraestructura berlinesa. Surgen planes de refacción barrial en el núcleo antiguo-este, nuevos barrios de alto standard constructivo y estético y gigantescos complejos de vivienda vertical servidos mediante grandes avenidas en el centro y en la periferia de la "ciudad-capital".

Este gran esfuerzo del gobierno central requiere de un amplio despliegue de recursos económico-financieros, industriales, técnicos y humanos y tiene un costo muy alto: la priorización del desarrollo en la urbe Berlín les quita recursos a las ciudades medianas y pequeñas como Dresde, Leipzig, Rostock, Magdeburgo, Frankfurt-Oder, etc., etc.

Las ciudades nombradas, así como decenas de otras padecen hasta hoy de un alarmante deterioro, especialmente las áreas de interés histórico afectadas por la guerra y las inclemencias del tiempo, ya que durante más de cuarenta años no obtuvieron la mantención requerida. 
Desde la Reunificación, el gobierno de la República Federal de Alemania realiza un esfuerzo sobrehumano para reparar infraestructura vial, sanitaria y constructiva dañada, para restaurar monumentos de interés histórico y sanear barrios postergados en la otrora Alemania, una tarea tanto descomunal como onerosa para el bolsillo de todos los alemanes, una magna actividad que puede ser denominada con razón "los chantiers más activos de Europa en la actualidad" (especialmente en Berlín oriental, Dresde y Leipzig).

En Cuba, los esfuerzos hacia la consolidación de la descentralización, de la regionalización territorial y demográfica han tenido un inusitado éxito, las cifras actuales hablan por sí mismas. En 1955, Cuba contaba en total con 6 millones de ciudadanos, y en La Habana habitaban 1,5 millones de personas. En 1995, cuarenta años más tarde, la Isla de Cuba cuenta con 11 millones y La Habana sólo con 2,2 millones de habitantes, una proporción demográfica óptima.

Sin embargo, este formidable esfuerzo hacia las regiones también tuvo su precio: pues fue alcanzado con la postergación del desarrollo progresivo de la capital La Habana, lo que se pretende corregir.

Es éste el trasfondo de las recientes actividades y nuevas iniciativas emprendidas por el Estado, por el Municipio de La Habana y decenas de instituciones especializadas integradas en torno al desarrollo de la Planificación Física de la Capital La Habana, formulación de estrategias de remodelación urbanas, reordenamiento y finalmente, el "Plan Maestro de La Habana Vieja".

\section{Presentación del Plan Maestro de La Habana Vieja ${ }^{1}$}

Desde hace algún tiempo, un grupo de profesionales de distintas especialidades, a instancias de la Oficina del Historiador de la Ciudad de La Habana y de la Agencia Española de Cooperación Internacional, AECI, trabaja en la elaboración del Plan Maestro de La Habana Vieja. El Plan Maestro pretende detectar los problemas actuales de La Habana Vieja y proponer para ellos soluciones técnicas, humanas y económicas, y al mismo tiempo, anticiparse a los del futuro, a fin de evitarlos.

\footnotetext{
${ }^{1}$ Extractos del tríptico ¿Qué es el Plan Maestro de La Habana Vieja?, Plan Maestro, Revitalización Integral de la Habana Vieja, Ed. Oficina del Historiador-Ciudad de La Habana, y Agencia Española de Cooperación Internacional, s/f. (en Evento presentación del Plan Maestro, realizado entre el 11 y el 14 de diciembre de 1995).
} 


\section{REVISTA DE \\ URBANISMO \\ http://revistaurbanismo.uchile.cl}

Manejo y gestión de áreas históricas. El Plan Maestro de La Habana Vieja 1995-96

Handling and management of historic areas. The Master Plan 1995-96 La Habana Vieja 1995-96

Los aspectos más destacados son:

- las condiciones de las viviendas, los edificios y los espacios exteriores e interiores, tanto en el aspecto de seguridad como en el de salubridad;

- los servicios para uso de la población residente en La Habana Vieja, y

- los programas necesarios para que:

a) La Habana sea un área básicamente residencial y lograr, al máximo posible, que sus habitantes no sean desplazados a otros sectores de la ciudad;

b) Ios edificios de La Habana Vieja sean consolidados y rehabilitados, al mismo tiempo que se construyan nuevas viviendas en los solares existentes;

c) los servicios de agua, saneamiento y electricidad tengan buen funcionamiento en el área.

Por otro lado, el Plan Maestro debe proyectarse hacia el futuro, y por ello establece en el territorio una serie de zonas diferenciadas, de manera que en unas se permitan usos turísticos, oficinas y servicios, con el básico residencial, mientras que en otros sea este último el único admisible.

Por último, se regulan las intervenciones en los edificios existentes y se dan normas para la arquitectura y la construcción de los nuevos, pues La Habana Vieja es un bien patrimonial del pueblo cubano y, como tal, se debe defender.

\section{Desarrollo urbano y problemas principales generados}

Como corolario de la situación proporcional alcanzada hacia 1995: La Habana cuenta con 2,2 millones de habitantes frente a 11 millones en el resto del país, ocupando el $1 \%$ de la superficie de la isla.

En 40 años, la capital sólo aumentó poblacionalmente en 700.000 habitantes. Sólo una cantidad relativamente menor de población se radica en la periferia urbana (despliegue estatal construyendo los Nuevos Conjuntos Residenciales del Este, el Conjunto Residencial Juegos Panamericanos, etc.). La mayor parte de los inmigrantes pasa a residir dentro de la ciudad, también o especialmente el barrio de La Habana Vieja, causando a la infaestructura existente, un proceso masivo de densificación. A diferencia de muchas urbes hispanoamericanas, en la periferia de La Habana no existen barrios marginales tugurizados. 


\section{Los desafíos}

Las bien intencionadas metas contenidas dentro del Plan Maestro enfrentan en el futuro desafíos legales, estructurales, de financiamiento, de gestión participativa, de impacto vehicular, etc.

Mucho ha sido dicho en La Habana acerca del manejo y la gestión de áreas históricas. Sin embargo, "el caso cubano", a diferencia de otros ejemplos, necesitará de un preámbulo de pasos complejos que deben ser resueltos, antes de encarar la gestión barrial propiamente tal.

\section{Zonificación de La Habana Vieja}

Partiendo con el planteamiento de la tesis de que el "modelo cubano" se encuentra en la transición hacia una "apertura" (ajuste sui generis socio-económico y político), múltiples impactos y efectos exógenos son de esperar en la ciudad, especialmente en su estructura e infraestructura portuaria y en su área comercial, la cual atenderá en el futuro con mayor énfasis actividades turísticas masivas (el turismo genera los mayores ingresos del país), de exportación, de importación y complementarias.

Además, es de esperar la generación de nuevas áreas comerciales y de abastecimiento, endógenas o locales, que ameritarán de una infraestructura específica y de un espacio propio.

En resumen, una vez finalizado el bloqueo, se desarrollarán áreas con su propia especialización funcional y con sus nuevas delimitaciones y de allí también surgirán los primeros problemas: dentro de un Barrio sumamente densificado con gran déficit de plazas y áreas útiles exteriores, y con calles estrechas, ¿de dónde sacar o generar espacio suplementario para acomodar nuevas funciones necesarias? (¿de dónde "reconquistar" funciones urbanas recreativas, turísticas, gastronómicas, áreas comerciales, financieras, de bodegaje, etc.?).

Complementario con lo anterior, ¿cómo se manejará el esperado "futuro impacto vehicular"?

La actual infraestructura vial barrial no está diseñada para enfrentar en corto tiempo el impacto e incremento vehicular que en otras urbes se desarrolló en años. Aún con un reordenamiento apropiado de tráfico, se carece de área de parqueo o estacionamiento necesario. El nivel freático alto, por otro lado, encarece enormemente la adecuación de área subterránea para el efecto. ¿Se optará acaso por erigir torres periféricas de parking, reservando el área interior solo para el servicio público, para la bicicleta individual, la bici y moto-taxi? 
Actualmente, en la Plaza Vieja, se está demoliendo el único parqueadero subterráneo existente (del tiempo de Batista) para reconvertirlo exclusivamente en "plaza recreativa" siguiendo un modelo "historicista". En barrios históricos de Berlín occidental se han realizado compromisos, aún con elevado costo financiero: se han construido parqueaderos subterráneos, "invisibles" al ojo ciudadano, mediante gruesas lozas sobre las cuales se ubican los parques recreativos conteniendo las áreas verdes correspondientes.

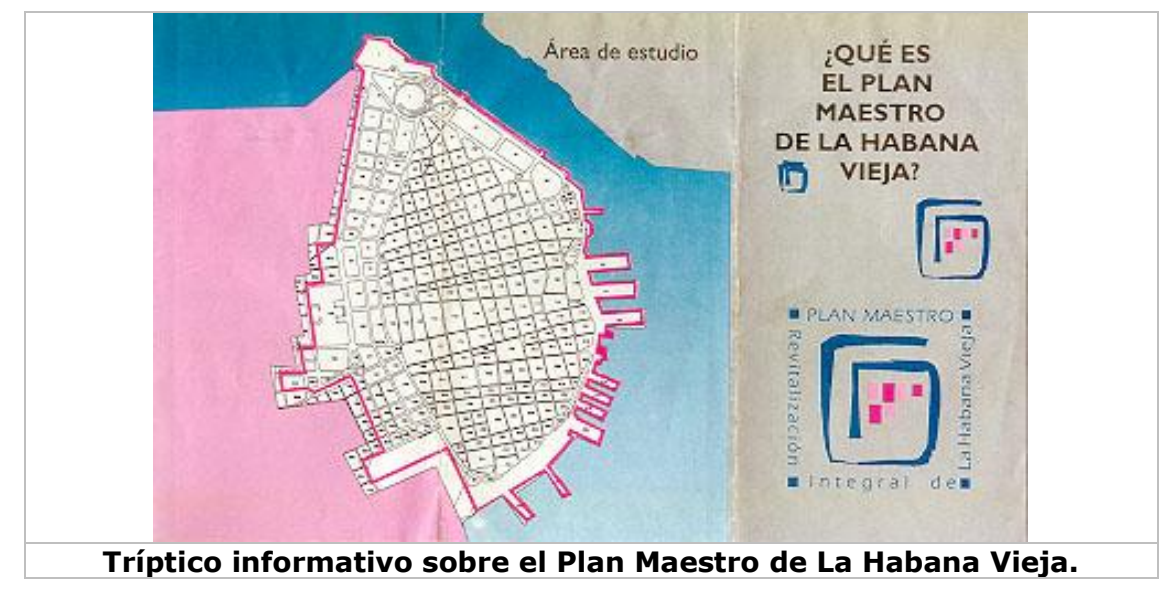

\section{¿Cómo generar recursos para financiar obras barriales?}

En lo referido al tema de la vivienda, desde los primeros años de la Revolución, la privatización "igualitaria" de vivienda por parte del Estado, y la Ley General de Vivienda, en 1986, dejaron problemas legales y económicos que atender:

En la mayoría de los Municipios occidentales, se recurre al "impuesto predial", un recurso propio para generar ingresos necesarios para la inversión barrial. El manejo se basa en el instrumento de los sistemas catastrales computarizados con los correspondientes "avalúos individuales" de predios y edificaciones.

Al mismo tiempo, los planes catastrales pormenorizados sirven de base para la planificación urbana. En La Habana, el manejo de este recurso requerirá de cambios y ajustes complementarios, ya que muchas de las prerrogativas usuales en otros países no existen, por ejemplo, actualmente, el valor intrínseco de algunas edificaciones es obviado, los precios por metro cuadrado de vivienda no obedecen al valor real, ya que aún no existe el libre mercado inmobiliario con sus regulaciones y parámetros específicos que se orientan hacia el tamaño/superficie, lo situacional y la calidad de los inmuebles. 
Con el triunfo de la Revolución, el tema de la propiedad queda definido, ya que los ocupantes o inquilinos de las viviendas pasan a ser los propietarios. En el año 1986, la Ley General de la Vivienda o "Ley de Reforma Urbana" consolida este status, dándoles a los residentes la oportunidad de pasar a ser propietarios valorando el tiempo que pagaron arrendamiento, más la diferencia faltante para cubrir la totalidad del valor de su vivienda. Los acreedores de vivienda nueva reciben un contrato a pagar en un plazo de 20 años.

El magnánimo Estado realiza esto en el espíritu de que los flamantes nuevos propietarios vayan a invertir en sus viviendas y se hagan cargo de su mantenimiento, sacándose "un peso de encima". Que esto no resultó así, lo atestigua entretanto el precario estado de las edificaciones antiguas del barrio Viejo de La Habana, por una simple razón: el mantenimiento de edificaciones antiguas, a veces palacios de valor histórico-cultural, es oneroso. Los modestos sueldos generalizados de los propietarios no alcanzan a cubrir inversiones que por otro lado ameritan para la restauración, de conocimientos técnicos y habilidades artesanales. Esto no significa que no se haya invertido o que no se hayan alcanzado logros en cuanto a mejoramiento de la calidad de standard de las viviendas.

Han existido dos modalidades de gestión o autogestión urbana de vivienda:

a) una fomentada directamente por el Estado: Ias micro "Brigadas Laborales", y

b) la modalidad de gestión o autogestión "privada".

La Gestión de la Micro-Brigada era una forma de acceder al alojamiento "por la vía laboral", es decir, se forman brigadas dentro de los centros de trabajo dedicados a la construcción de vivienda con el fin de restaurar edificaciones ruinosas. Luego, dentro de una asamblea, se distribuían las viviendas terminadas a los colegas más necesitados y a los de la fábrica. Este modelo fue criticado con razón, por ser exclusivista, es decir, no favorecía a gente necesitada que no tuviera vinculación directa con estos centros de trabajo. Por lo tanto, se buscó otro mecanismo de acceso al hábitat, cambiándose las "Brigadas Laborales" por las "Brigadas Sociales", que a diferencia de las anteriores se dedicaron a sanear áreas insalubres con criterio social de saneamiento más integral (erradicando "ciudadelas", "cuarterías" o áreas tugurizadas).

En cuanto a la gestión o autogestión "privada" ó, el "esfuerzo propio", los dueños de un inmueble podían explotar comercialmente los departamentos contenidos en él mediante dos variantes: renta en "inquilinato", o venta.

Sin realizar inversiones, se mantenía la vivienda "en cuartería", es decir, habitaciones sin baño o cocina individual, generalmente edificios de hasta cuatro pisos con pasillos 
REVISTA DE

$\bigcup_{15 S \mathrm{R} N \text { 0717.5051 }}$ ANISMO

http://revistaurbanismo.uchile.cl
Manejo y gestión de áreas históricas. El Plan Maestro de La Habana Vieja 1995-96

Handling and management of historic areas. The Master Plan 1995-96 La Habana Vieja 1995-96

interiores que dan hacia un patio de luz central. En la planta baja se hallan situados los servicios higiénicos y las cocinas colectivas. En una segunda etapa, el propietario decide convertir "la cuartería" en departamentos, dotando de baños y cocinas a las dependencias individuales. Los aposentos sobrepasan los 4 metros de altura, lo que ha posibilitado la modalidad de construir un entrepiso dentro de los pequeños departamentos, llamado la "barbacoa", que duplica el espacio útil. Mediante la habilitación de dormitorio en el altillo, quedando abajo el estar y el comedor al lado de la cocina y el baño con ducha, los departamentos logran un standard modesto, pero muy superior al de la "cuartería".

Con la Ley de Reforma Urbana, mediante disposición transitoria dentro de la citada Ley, los inquilinos que vivían en viviendas consideradas como "precarias", no necesitaban pagar arriendo, ya que se daban en régimen de usufructo gratuito, mientras no contaran con los servicios necesarios y con las condiciones sanitarias y un standard mínimo de habitabilidad indicados por la ley. Al existir usufructo gratuito, los inquilinos ya no cuentan con un propietario que los controla o que impide en el peor de los casos que se intervenga en los espacios.

Los nuevos propietarios intervienen en autogestión mejorando la calidad de las viviendas. La modalidad descrita adolece de la calidad técnica requerida para este tipo de refacciones constructivas, ya que la asistencia técnica por parte del Municipio ha sido escasa, lo que ha tenido como resultado un gran número de "soluciones habitacionales anárquicas", contaminación parcial de la Bahía debido a evacuaciones directas de heces, problemas de estabilidad debido a barbacoas mal construidas y otros.

Sin embargo, los logros positivos son los más, considerándose que hasta ahora, el $80 \%$ de viviendas con usufructo gratuito han alcanzado mejoras en su grado de habitabilidad. Pero, a pesar de todos los esfuerzos desplegados hasta ahora, tanto públicos como privados, no han sido capaces de satisfacer la enorme demanda existente para la restauración de tipología doméstica, pública y comercial (¿rehabilitación participativa subsidiada?).

El gran reto consiste desde ya en reunir e integrar todos los recursos disponibles para el logro de una metodología de Estrategias de Rehabilitación Integral Urbana, dentro de la cual la tipología doméstica requerirá del mayor esfuerzo, tanto debido al aspecto cuantitativo como a las limitantes financieras de este sector frente a la tipología comercial y pública.

\section{Capacidad de gestión: dinámica participativa}

Obviamente, la experiencia anteriormente enunciada propone por lógica, el desarrollo de metodologías de gestión urbanas participativas, en las cuales el sujeto social está directamente involucrado en la toma de decisiones concerniente a sus intereses comunitarios y personales. Sin embargo, la experiencia asimismo no nos recomienda 


\section{REVISTA DE

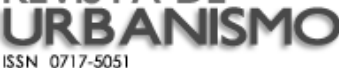

http://revistaurbanismo.uchile.cl
Manejo y gestión de áreas históricas. El Plan Maestro de La Habana Vieja 1995-96

Handling and management of historic areas. The Master Plan 1995-96 La Habana Vieja 1995-96

una autogestión individualista, sino un proceso barrial comunitario enmarcado "dentro de una infraestructura de apoyo técnico, administrativo y financiero". Dentro de esta infraestructura, los actores debieran tener origen mixto interdisciplinario, interviniendo entes integrados Municipales, Universitarios, ONG's y otros, junto a los grupos metas comprometidos.

Por medio del esfuerzo propio de la población, históricamente se han construido más viviendas en Cuba que las producidas por el Estado directamente..., si bien en dicho impacto de producción popular no estuvo ajeno el Estado, garante de servicios sociales esenciales, suelo y recursos materiales, faltó el desarrollo de modelos de organización social más eficaces, no hubo la asesoría técnica que requieren estos procesos al nivel urbano, para que resulten más efectivos, así como han sido excepcionales los casos en que se implementaron métodos participativos. El esfuerzo propio de la población en realidad se ha caracterizado por ser un proceso de enfrentamiento individual por cada familia de sus problemas, principio distante de la cultura de cooperación y hermandad fomentada en la población cubana.

... Muchas veces se ha planteado si la puesta en práctica de experiencia de ayuda mutua o cooperativas no representarían una mayor eficiencia de la mano de obra popular, un mayor aprovechamiento de los recursos materiales al poderse proyectar conjuntos en vez de casas aisladas, una mayor optimización del suelo urbanizable y posiblemente la perdurabilidad de relaciones vecinales más sólidas constatables en un mejor cuidado del inmueble y el entorno urbano... ${ }^{2}$.

\section{Planos}

(Vid Infra)

\footnotetext{
2 MESIAS GONZÁLEZ, Rosendo, Arqto. Coordinador, "Tecnologías para la producción y rehabilitación del habitat participativo y autogestionable, Primera Parte", Seminario Iberoamericano auspiciado por Red XIV. b - CYTED: "Viviendo y Construyendo", Red "Viviendo y Construyendo" - Cuba, Instituto de Planificación Física, Habana Vieja, 24 al 26 de noviembre de 1994. Víctor S. Pelli, Héctor Cuervo, Salvador Gomila, Edín Martínez, Rafael Rueda, Selma Díaz, Milagros cabrera, Mario Coyula. Colección Enfoques.
} 


\section{REVISTA DE $\bigcup_{15 S \mathrm{~N} N \text { 0717-5051 }} \mathrm{BN}$ ISMO}

http://revistaurbanismo.uchile.cl
Manejo y gestion de áreas historicas. El Plan Maestro de La Habana Vieja 1995-96

Handling and management of historic areas. The Master Plan 1995-96 La Habana Vieja 1995-96

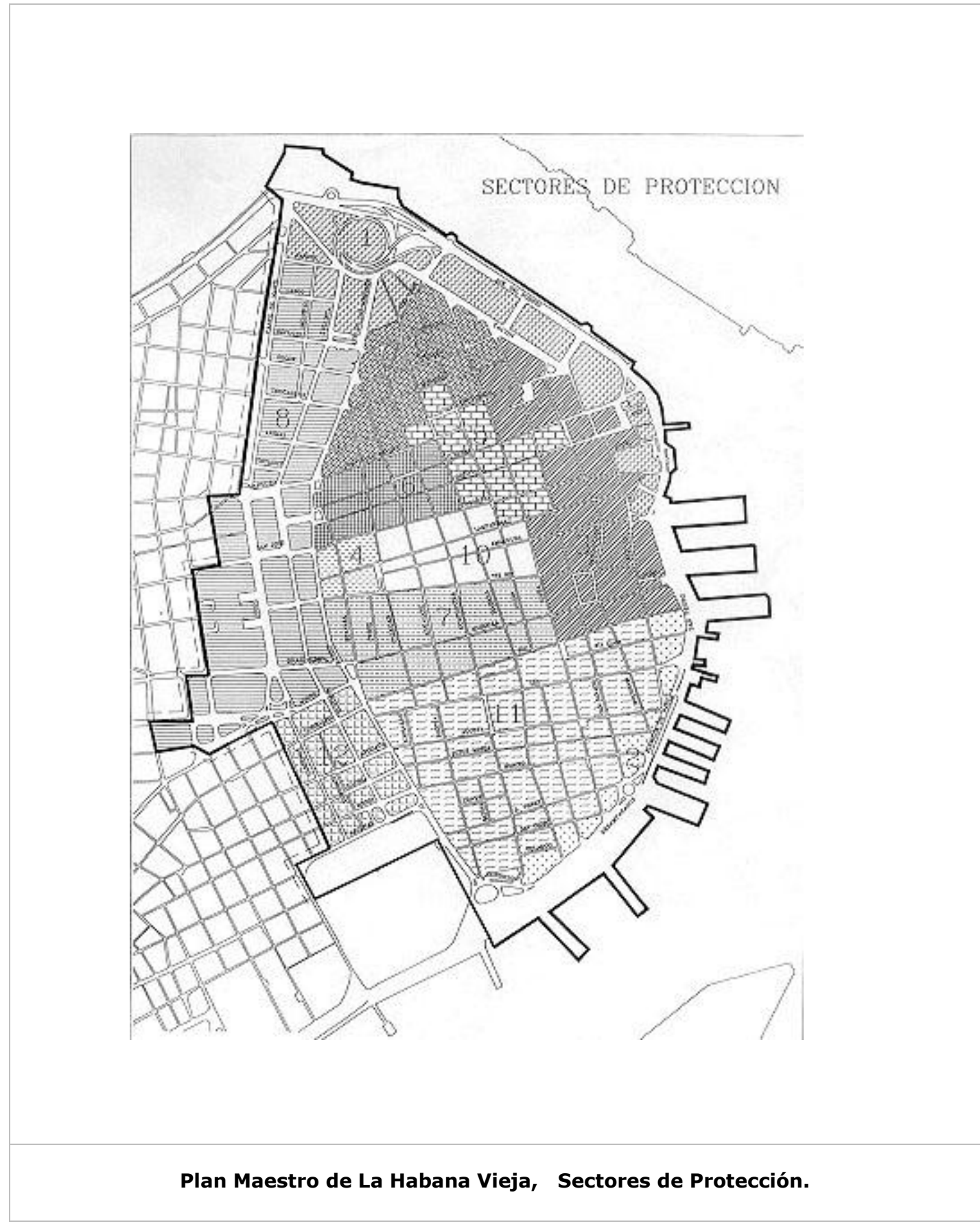




\section{REVISTA DE $\bigcup_{15 S \mathrm{R} N}^{0777-5051}$ ANISMO}

http://revistaurbanismo.uchile.cl
Manejo y gestion de áreas históricas. El Plan Maestro de La Habana Vieja 1995-96

Handling and management of historic areas. The Master Plan 1995-96 La Habana Vieja 1995-96

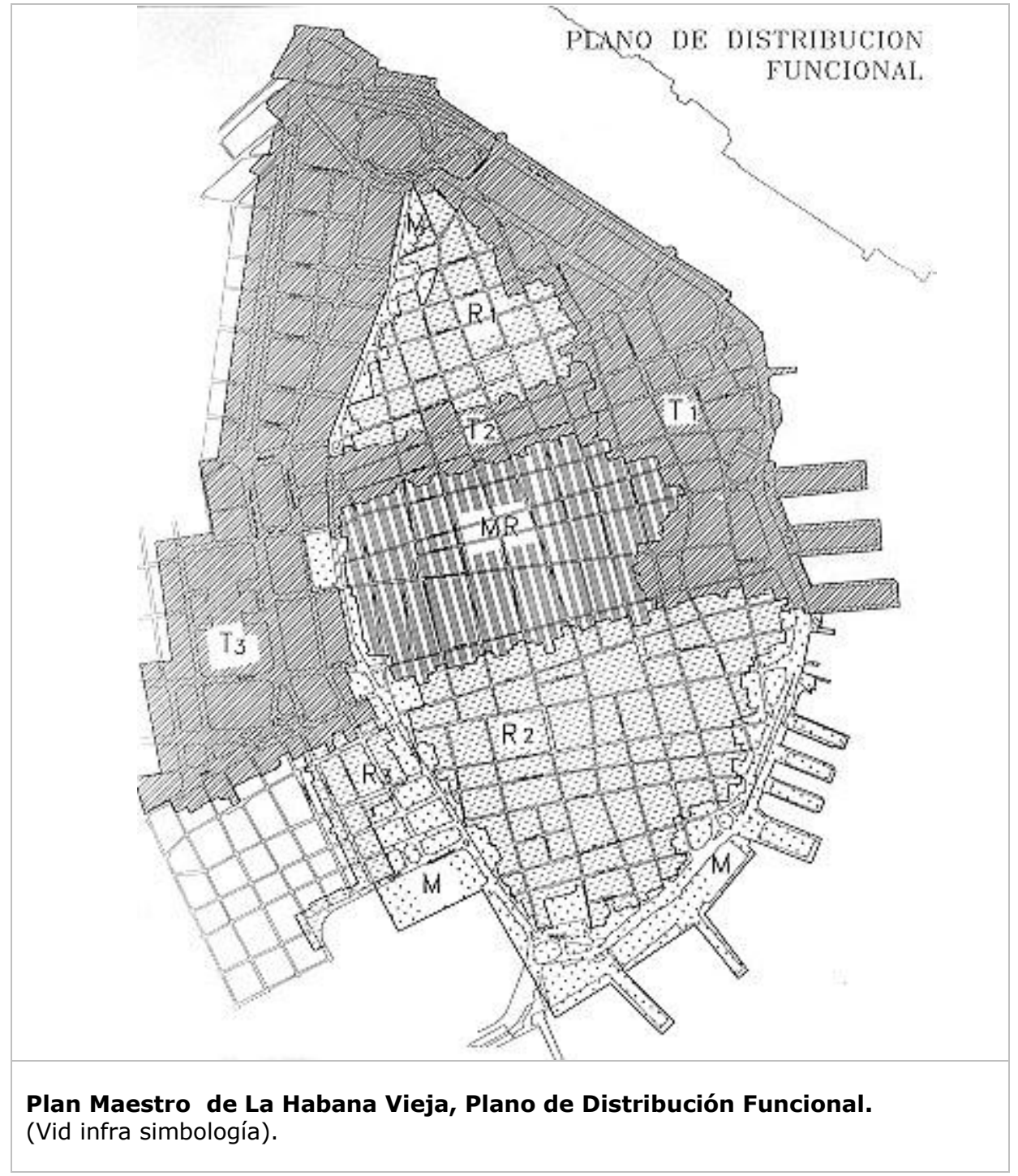




\section{REVISTA DE $\bigcup_{\text {ISSN }}$ R717-5051 $A N I S M O$

http://revistaurbanismo.uchile.cl

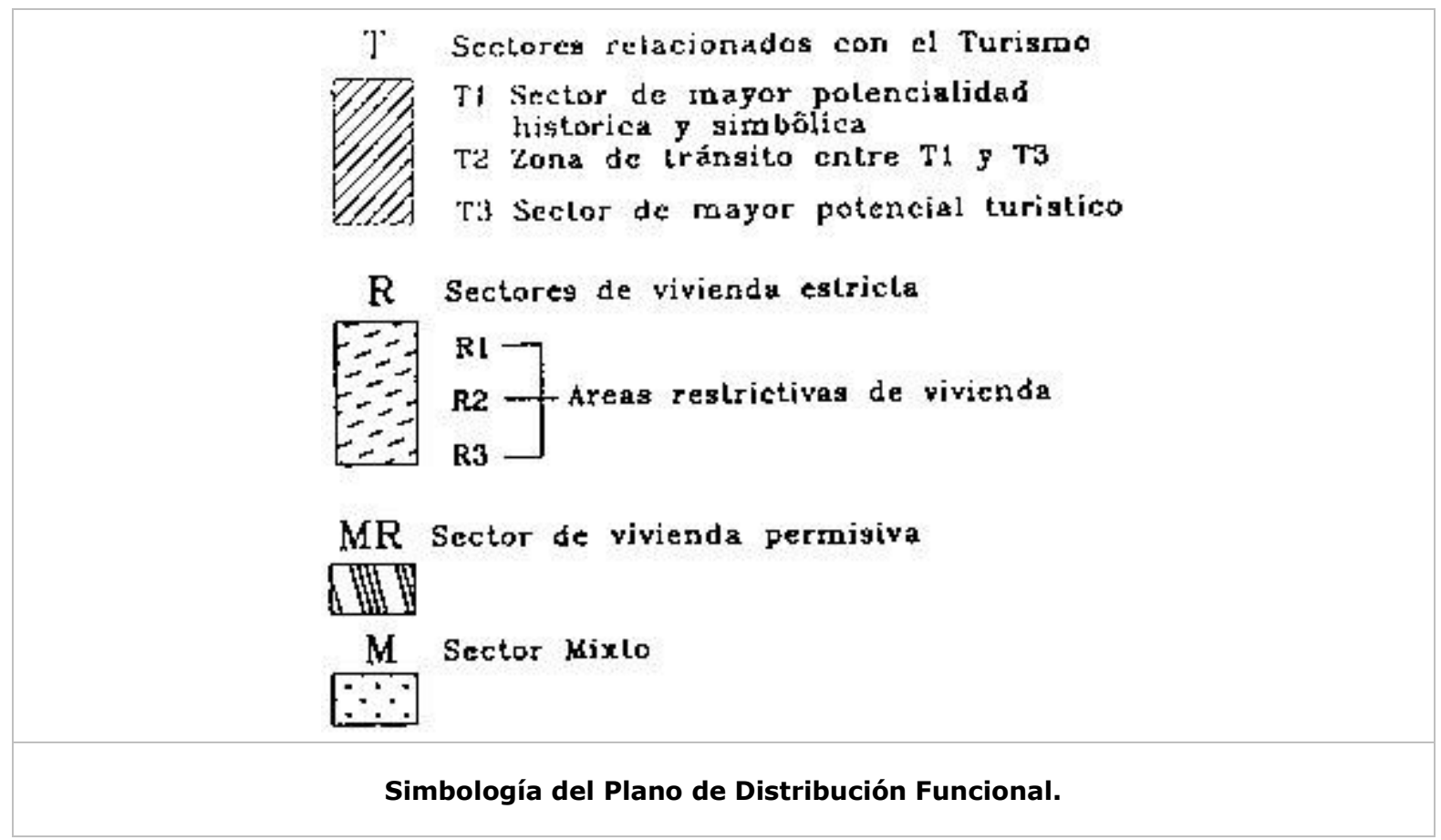

Nota del autor: Last but not least, quisiéramos destacar y agradecer el apreciable apoyo técnico profesional y sobre todo, informativo dentro del ámbito cubano, recibido por parte del Arqto. Rosendo MESÍAS G., tanto en Quito como en La Habana. 